POS $\quad$ PROCEEDINGS

\title{
Nonrelativistic energy levels of helium atom
}

\section{D.T. Aznabayev*}

Bogoliubov Laboratory of Theoretical Physics, Joint Institute for Nuclear Research, Dubna 141980, Russia

The Institute of Nuclear Physics, Ministry of Energy of the Republic of Kazakhstan, 050032 Almaty, Kazakhstan

L.N. Gumilyov Eurasian National University, 010000 Astana, Kazakhstan

E-mail: aznabaevetheor.jinr.ru

\section{A.K. Bekbaev}

Bogoliubov Laboratory of Theoretical Physics, Joint Institute for Nuclear Research, Dubna 141980, Russia

Al-Farabi Kazakh National University, 050038 Almaty, Kazakhstan

E-mail: bekbaev-askhatemail.ru

\section{Vladimir I. Korobov}

Bogoliubov Laboratory of Theoretical Physics, Joint Institute for Nuclear Research, Dubna 141980, Russia

Peoples' Friendship University of Russia (RUDN University), 6 Miklukho-Maklaya St, Moscow, 117198, Russia

E-mail: korobov@theor.jinr.ru

The nonrelativistic energy levels of a helium atom are calculated for $S, P, D$ and $F$ states. The calculations are based on the variational method of "exponential" expansion. The convergence of the calculated energy levels is studied as a function of the number of basis functions $N$. This allows us to claim that the obtained energy values (including the values for the states with a nonzero angular momentum) are accurate up to 28-35 significant digits.

International Conference on Precision Physics and Fundamental Physical Constants - FFK2019

9-14 June, 2019

Tihany, Hungary

* Speaker. 


\section{Introduction}

The quantum problem of three bodies with Coulomb interaction is one of the most notable nonintegrable problems in quantum mechanics. At the same time, extremely accurate numerical solutions for the problem of bound states for a system of three particles may be obtained with modern computers. For example, the nonrelativistic energy of the ground state of helium with a nucleus of an infinite mass is now known accurately to 46 significant digits [1].

In the present study, a version of the variational method (the so called "exponential" expansion) [2] that allows to numerically solve the quantum Coulomb three-body bound state problem with a very high precision, which is easily applicable as well to the states with a nonzero angular momentum, is considered. This method is used to calculate the nonrelativistic energies of a helium atom for $S, P, D$, and $F$ states. It is shown that the developed method is an efficient and flexible instrument for studying Coulomb systems. An analysis of convergence proves that the method is highly accurate and demonstrates that nonrelativistic energies accurate up to 28-35 significant digits may be obtained with rather moderate efforts.

Developing of such high precision methods is of importance for the reason that it may help solving a wide variety of problems that are of interest in physics. For example, antiprotonic helium atoms are of particular interest, which allows for high precision studies of energy spectrum of this exotic system and inferring of various properties of an antiproton from comparison of theory and experiment $[3,4]$. Here it is worthy to mention a recent interest to the antiprotonic helium as a tool for constrains on various fifth forces $[5,6]$ to set general limits on new interactions beyond the Standard Model.

\section{Generalized Hylleraas expansion}

Let us consider the generalized Hylleraas expansion for the states of arbitrary total orbital momentum $L$ [7]:

$$
\psi\left(r_{1}, r_{2}\right)=\sum_{l_{1}+l_{2}=\mathscr{L}} \mathscr{Y}_{L M}^{l_{1} l_{2}}\left(r_{1}, r_{2}\right)\left[e^{-\alpha r_{1}-\beta r_{2}-\gamma r_{12}} \sum_{l, m, n \geq 0} C_{l m n} r_{1}^{l} r_{2}^{m} r_{12}^{n}\right]
$$

$\mathscr{L}=L$ for the states of "normal" spatial parity $\Pi=(-1)^{L}$, and $\mathscr{L}=L+1$ for the states of "anomalous" spatial parity $\Pi=(-1)^{L+1}$. The complex parameters in the exponent are generated in a pseudorandom way. The $\mathscr{Y}_{L M}^{l_{1} l_{2}}$ functions are regular bipolar spherical harmonics [8] that depend on two angular coordinates:

$$
\mathscr{Y}_{L M}^{l_{1} l_{2}}\left(r_{1} r_{2}\right)=r_{1}^{l_{1}} r_{2}^{l_{2}}\left\{Y_{l_{1}}\left(\hat{r}_{1}\right) \otimes Y_{l_{2}}\left(\hat{r}_{2}\right)\right\}_{L M},
$$

where $Y_{l}(\hat{r})=Y_{l m}(\theta, \varphi)=\sqrt{\frac{2 l+1}{4 \pi} \frac{(l-m) !}{(l+m) !}} P_{l}^{m}(\cos (\theta)) e^{i m \varphi}, P_{l}^{m}(\cos (\theta)$ are associated Legendre polynomials. Spatial parity operator $P \psi=\pi \psi$ acts on the spatial coordinates in the following way: $P\left(r_{1}, r_{2}\right) \rightarrow\left(-r_{1},-r_{2}\right)$. The ease of use of the $\mathscr{Y}_{L M}^{l_{1} l_{2}}$ functions stems from the fact that they correctly reproduce the behavior of the wave function at $r_{1} \rightarrow 0$ (or $r_{2} \rightarrow 0$ ), and retain the reasonable requirement of boundedness of the function within the domain of variables for the expression within square brackets in Eq. (2.1). 
Table 1: Convergence of the nonrelativistic energy of the ground state of a helium atom.

\begin{tabular}{cl}
\hline \hline Basis $(N)$ & $E_{n r}$ \\
\hline 10000 & -2.9037243770341195983111592451939 \\
14000 & -2.903724377034119598311159245194398 \\
18000 & -2.90372437703411959831115924519440432 \\
22000 & -2.90372437703411959831115924519440443 \\
\hline \hline
\end{tabular}

\section{Results and discussion}

In Table 1 we check the convergence of energy for the ground state of helium versus increasing basis sets of the variational expansion. The structure of "layers" of basis functions is very similar to what was used in our previous calculations [9], where it was explicitly published (see Table I in [9]). In present case we optimized the variational basis with $N=10000$ functions and 8 layers. For the final calculation with $N=22000$ functions we used 12 layers, and for the last four layers the ends of intervals $\left[A_{1}, A_{2}\right]$ and $\left[B_{1}, B_{2}\right]$ grew exponentially: $A_{1}(j)=B_{1}(j)=10^{j-4}, A_{2}(j)=B_{2}(j)=10^{j-3}$ for $j=9, \ldots, 12$. Computations were performed in the duodecimal arithmetics (about 100 decimal digits). Programs of duodecimal precision were developed by our group in order to overcome the problem of the numerical instability of calculations at large values of $N$.

Results of numerical calculations of the nonrelativistic energies for $S, P D$, and $F$ states of a helium atom are presented in Table 2. Variational parameters were optimized manually. It should be noted that the optimal variational parameters for different states differ significantly, and the calculation accuracy depends to a considerable extent (5-8 digits) on the particular choice of optimal variational parameters for a given bound state. Basis sets with $N=10000$ functions were used to optimize the variational parameters. When the non $S$ states listed in the table were calculated, 4 to 6 "layers" of basis functions were used, while for the $S$ states calculations were done in the similar way as for the ground state. The results in Table 2 are presented for two subsequent calculations with increasing basis sets, what allows to demonstrate convergent digits. The third line shows the results of variational calculations by Drake and Yan [10] performed in year 1992, where the Rydberg states (excluding $S$ states) of helium were studied. Comparison between two calculations demonstrates excellent agreement. The largest set for each particular state has been chosen by the reason that further increase of the basis gives rise to numerical instability of calculations within given duodecimal arithmetics. As may be seen numerical precision for triplet states is slightly higher, probably that is due to smaller effect of the logarithmic singularity. For higher orbital angular momentum states we have managed to achieve precision of 27-28 digits. Still that is the best known data for these states. All the calculations were performed on the Linux mainframe computers of our Laboratory.

For the ground state energy we compare our best obtained value with previously published results in Table 3. Indeed, explicit inclusion of the logarithmic singularity into a variational expansion may seriously improve precision of the results. On the other hand, with our variational basis function we can easily extend calculations to the states with excited electronic orbital as well as 
Table 2: Convergence of the nonrelativistic energies of the $S, P, D$, and $F$ states of a helium atom. $N$ is the number of basis functions. The two lines represent two consecutive calculations with the largest basis sets to show convergent digits. The third line presents calculations by Drake and Yan [10].

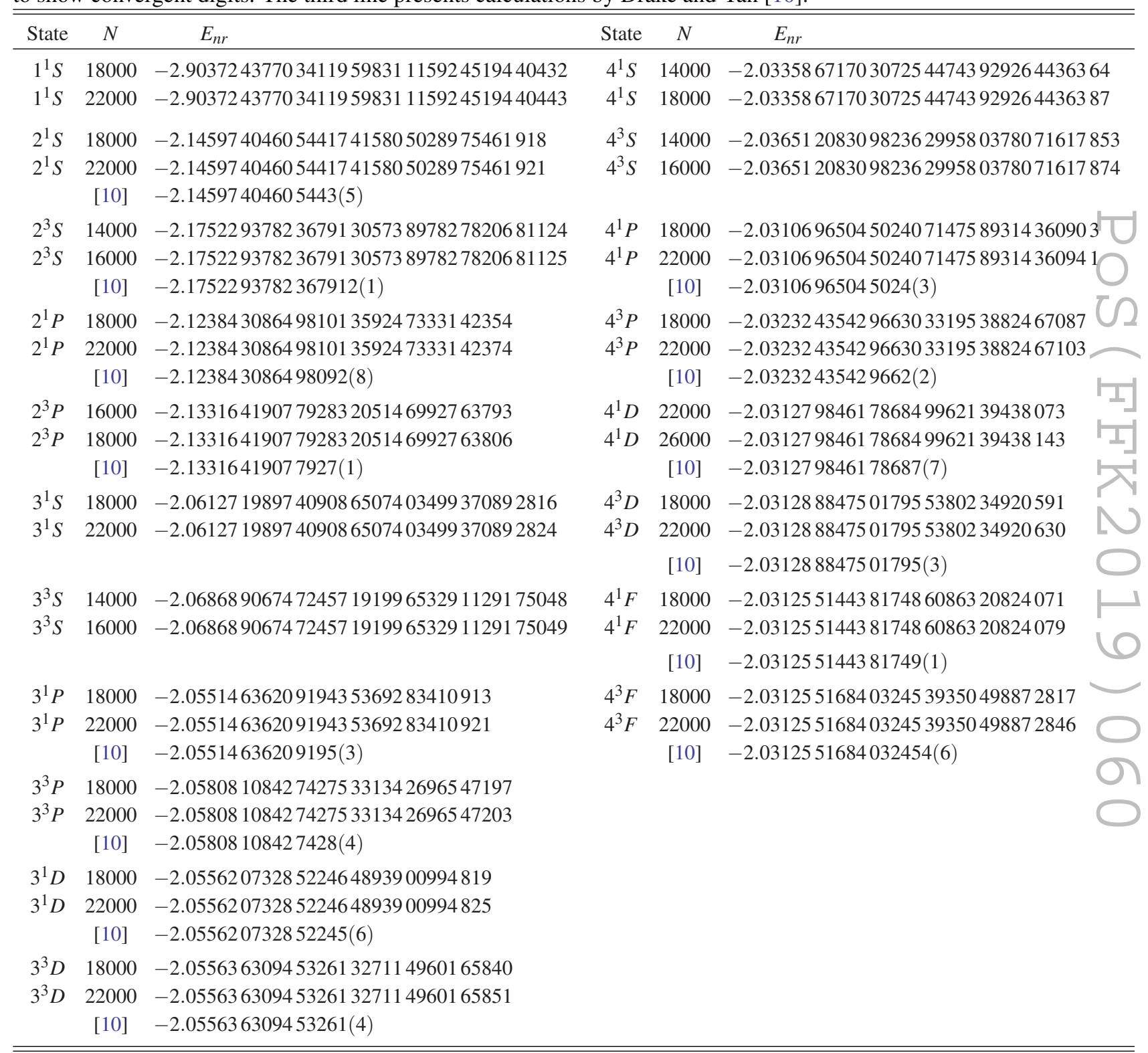

nonzero angular momentum states with large $L$.

Variational wave functions of bound states are obtained by solving the Schrodinger equation for the quantum three-body problem with Coulomb interaction using a variational approach based on exponential expansion with the parameters of exponents being chosen in a pseudorandom way. The results of these studies demonstrated that the energy values were accurate to 27-35 significant digits. 
Table 3: Comparison of the nonrelativistic energies of the ground state of a helium atom.

\begin{tabular}{lcrl}
\hline \hline Author (year) & Ref. & \multicolumn{1}{c}{$N$} & Energy (in a.u.) \\
\hline Drake et al. (2002) & {$[11]$} & 2358 & -2.903724377034119598311 \\
Korobov (2002) & {$[9]$} & 5200 & -2.903724377034119598311159 \\
Schwartz (2006) & {$[1]$} & 24099 & -2.903724377034119598311159245194404446696925310 \\
Nakashima, Nakatsuji (2007) & {$[12]$} & 22709 & -2.9037243770341195983111592451944044466969 \\
this work & {$[2]$} & 22000 & -2.90372437703411959831115924519440443 \\
\hline \hline
\end{tabular}

\section{Acknowledgements}

The work was supported by the Ministry of Education and Science Republic of Kazakhstan under grant IRN AP05132978, V.I.K. acknowledges support of the "RUDN University Program 5-100".

\section{References}

[1] C. Schwartz, Experiment and theory in computations of the He atom ground state. Int. J. Mod. Phys. E 15, 877 (2006); C. Schwartz, Further Computations of the He atom ground state. ArXiv:math-phys/0605018, (2006).

[2] D.T. Aznabayev, A.K. Bekbaev, V.I. Korobov, Nonrelativistic energy levels of helium atoms. Phys. Rev. A 98, 012510 (2018).

[3] M. Hori, A. Sótér, D. Barna, A. Dax, R. Hayano, S. Friedreich, B. Juhász, Th. Pask, E. Widmann, D. Horváth, L. Venturelli, and N. Zurlo, Two-photon laser spectroscopy of antiprotonic helium and the antiproton to electron mass ratio. Nature 475, 484 (2011).

[4] V.I. Korobov, Bethe logarithm for resonant states: Antiprotonic helium. Phys. Rev. A 89, 014501 (2014).

[5] E.J. Salumbides, W. Ubachs, and V.I. Korobov, Bounds on fifth forces at the sub-Ålength scale, J. Mol. Spectrosc. 300, 65 (2014).

[6] F. Ficek, P. Fadeev, V.V. Flambaum, D.F.J. Kimball, M.G. Kozlov, Y.V. Stadnik, and D. Budker, Constraints on Exotic Spin-Dependent Interactions Between Matter and Antimatter from Antiprotonic Helium Spectroscopy, Phys. Rev. Lett. 120, 183002 (2018).

[7] G.W.F. Drake, Angular integrals and radial recurrence relations for two-electron matrix elements in Hylleraas coordinates, Phys. Rev. A 18, 820 (1978).

[8] D.A. Varshalovich, A.N. Moskalev and V.K. Khersonskii, Quantum Theory of Angular Momentum (Nauka, Leningrad, 1975; World Scientific, Singapore, 1988).

[9] V.I. Korobov, Nonrelativistic ionization energy for the helium ground state. Phys. Rev. A 66, 024501 (2002).

[10] G.W.F. Drake and Zong-Chao Yan, Energies and relativistic corrections for the Rydberg states of helium: Variational results and asymptotic analysis. Phys. Rev. A 46, 2378 (1992).

[11] G.W.F. Drake, M.M. Cassar, and R.A. Nistor, Ground-state energies for helium, $\mathrm{H}^{-}$, and $\mathrm{Ps}^{-}$Phys. Rev. A 65, 054501 (2002).

[12] H. Nakashima and H. Nakatsuji, Solving the Schrödinger equation for helium atom and its isoelectronic ions with the free iterative complement interaction (ICI) method. J. Chem. Phys. 127, 224104 (2007). 